ARTICLE

\title{
Axon degeneration induces glial responses through Draper-TRAF4-JNK signalling
}

Tsai-Yi Lu ${ }^{1} \uparrow$, Jennifer M. MacDonald ${ }^{1}$, Lukas J. Neukomm¹, Amy E. Sheehan ${ }^{1}$, Rachel Bradshaw ${ }^{1}$, Mary A. Logan ${ }^{1, \dagger}$ $\&$ Marc R. Freeman ${ }^{1, \dagger}$

Draper/Ced-1/MEGF-10 is an engulfment receptor that promotes clearance of cellular debris in C. elegans, Drosophila and mammals. Draper signals through an evolutionarily conserved Src family kinase cascade to drive cytoskeletal rearrangements and target engulfment through Rac1. Glia also alter gene expression patterns in response to axonal injury but pathways mediating these responses are poorly defined. We show Draper is cell autonomously required for glial activation of transcriptional reporters after axonal injury. We identify TNF receptor associated factor 4 (TRAF4) as a novel Draper binding partner that is required for reporter activation and phagocytosis of axonal debris. TRAF4 and misshapen (MSN) act downstream of Draper to activate c-Jun N-terminal kinase (JNK) signalling in glia, resulting in changes in transcriptional reporters that are dependent on Drosophila AP-1 (dAP-1) and STAT92E. Our data argue injury signals received by Draper at the membrane are important regulators of downstream transcriptional responses in reactive glia.

\footnotetext{
${ }^{1}$ Department of Neurobiology, University of Massachusetts Medical School, Worcester, Massachusetts 01605, USA. † Present addresses: Solomon H. Snyder Department of Neuroscience, Johns Hopkins University School of Medicine, 725 N. Wolfe St., WBSB 1001, Baltimore, Maryland 21205, USA (T.-Y.L.); Junger's Center for Neurosciences Research, Oregon Health and Science University, 3181 SW Sam Jackson Park Rd., Portland, Oregon 97239, USA (M.A.L.); Vollum Institute, Oregon Health and Science University, 3181 SW Sam Jackson Park Rd., Portland, Oregon 97239, USA (M.R.F.). Correspondence and requests for materials should be addressed to M.R.F. (email: freemmar@ohsu.edu).
} 
G lial cells are exquisitely sensitive to perturbations of nervous system physiology $y^{1-7}$. In response to neural injury they coordinate a sophisticated cascade of reactive events, including engagement of phagocytic programmes to clear cellular debris, and secretion growth factors to recruit additional immune cells or promote neuronal survival and repair ${ }^{8}$. Reactive glial responses are driven in important ways by changes in glial gene expression following trauma. Strong upregulation of expression of GFAP and $S 100 \beta$ are among the earliest detectable changes in mammalian neural tissues after brain injury, and similar upregulation occurs in chronic neurodegenerative disease ${ }^{9,10}$. In peripheral nerves early events include the activation of JNK signalling, which is required for efficient Schwann cell de-differentiation and initiation of regenerative responses ${ }^{11}$. Initiating events in glial reactive responses are presumably driven by 'injury signals' released by damaged cells that signal to transmembrane receptors on glia, but whether and how cellular debris activates glial transcriptional responses remains mechanistically unclear.

Drosophila glia rapidly respond at the transcriptional level to axotomy, which argues reactive glial responses are an ancient feature of the metazoan nervous system ${ }^{12}$. Transcriptional reporters for the sole Drosophila STAT molecule, STAT92E, are induced in glial cells within hours after local axons are severed ${ }^{6}$. Axotomy also activates the glial JNK signalling cascade and ultimately transcriptional reporters for the Drosophila AP-1 complex (dAP-1) ${ }^{7}$. Activation of STAT92E-dependent transcriptional responses has been shown to require the engulfment receptor Draper ${ }^{6}$, suggesting STAT92E lies genetically downstream of Draper, although how axon degeneration is mechanistically linked to STAT92E- or dAP-1-mediated transcriptional changes is not known.

\section{Results}

Axon degeneration activates dAP-1 reporters in glia. To understand how axonal injury leads to glial transcriptional changes we sought to define the mechanisms regulating activation of the dAP-1 complex in glia after axotomy. We used the dAP-1 transcriptional reporters containing multiple tetradecanoylphorbol acetate response elements (TRE, that is the dAP-1 binding sequence) upstream of either eGFP (TRE-eGFP) or RFP $(T R E-R F P)^{13}$. Axon injury was induced by surgical removal of adult Drosophila third antennal segments, which severs $\sim 90 \%$ of olfactory receptor neurons (ORNs) projecting into the Drosophila antennal lobe and axon fragmentation begins within $\sim 12 \mathrm{~h}$ by activation of Wallerian degeneration ${ }^{2}$. Prior to axonal injury TRE-RFP was not detectable at significant levels in control animals in any part of the adult brain. However, one day after axonal injury (1 dpant, 1 day post antennal ablation), we observed strong activation of TRE-RFP throughout the brain (Supplementary Fig. 1), as previously observed for TRE-eGFP (ref. 7), along with glial hypertrophy and increased Draper levels in glia adjacent to the injury site. To determine whether activation of TRE-RFP represented a glial response to axon degeneration, we blocked ORN axon degeneration using highwire (hiw) mutants ${ }^{14}$. Glial cells in hiw ${ }^{\Delta N}$ mutant animals exhibited no morphological changes after axonal injury, TRE-RFP activation was not observed in any part of the brain, and Draper levels adjacent to the injury site remained similar to the uninjured controls (Fig. 1a). Hiw is not expressed at detectable levels in glia but is prominent in neurons (Fig. 1b), suggesting the lack of glial activation after axotomy in hiw mutants resulted from blockade of axon degeneration, rather than a cell autonomous role for hiw in glial activation. Consistent with this notion, pan-neuronal expression of Highwire in $h i w^{\Delta N}$ mutant background rescued glial response to axon degeneration (Supplementary Fig. 2b) while glial expression of a hiw cDNA in the hiw ${ }^{\Delta N}$ mutant background did not lead to activation of the TRE-RFP reporter (Supplementary Fig. 2a). Activation of the Wallerian degeneration signalling pathway is therefore essential for brain-wide induction of dAP-1 transcriptional reporters in glia after nerve injury. Furthermore, these data indicate glial cells respond specifically to signals generated by degenerating axons, rather than other alterations in axons caused by axotomy (for example loss of neuronal activity).

Glia activate dAP-1 reporters after injury through Draper. The engulfment receptor Draper activates glial phagocytic function during Wallerian degeneration ${ }^{2}$. We found draper ${ }^{45}$ null mutant animals also exhibited a striking loss of TRE-eGFP induction 1 day after axon injury compared to controls (Fig. 1c,d), indicating that Draper is also required for dAP-1 reporter activation in response to axonal degeneration. ORN axon terminals only project to the antennal lobe. The lack of TRE-eGFP activation in draper mutants observed throughout the adult brain implied that Draper was required for TRE-eGFP activation even in glia at sites distant from degenerating axons in the antennal lobe. To characterize the autonomy of Draper function in activation of dAP-1 transcriptional reporters in brain glia, we generated draper ${ }^{45}$ mutant clones using the mosaic analysis with a repressible cell marker (MARCM) approach ${ }^{15}$, severed antennal ORN axons, and assayed TRE-RFP activation 1 day after axotomy. Based on morphological analysis of clones we determined that activation of TRE-RFP occurred in ensheathing (Fig. 1e) and cortex glia (Fig. 1f), but not in astrocytes (Supplementary Fig. 3), consistent with a previous study demonstrating Draper is expressed solely in these glial subtypes in the Drosophila adult brain ${ }^{16}$. While control clones in each subtype of glial cell showed strong activation of TRE-RFP in response to axonal injury, draper ${ }^{45}$ mutant clones did not activate the TRE-RFP reporter (Fig. 1e,f). This was even true when rraper $^{45}$ mutant clones were made in cortex glia, which are not in direct contact with severed axons (for example Fig. 1f). This was surprising in light of the fact that Draper/Ced-1 is considered a recognition receptor for 'eat me' cues on the surface of engulfment targets such as axonal debris ${ }^{1}$, which we presumed would require cell-cell contact. This observation suggests that Draper may be activated by a soluble ligand released by severed ORNs, or alternatively that Draper-dependent glial-glial signalling may occur in cortex glia upstream of dAP-1 reporter activation, perhaps similar to MEGF10 signalling in starburst amacrine cells during tiling where MEGF10 appears to act genetically as both a receptor and ligand ${ }^{17}$.

TRAF4 signals downstream of Draper after axonal injury. Based on the potent regulation of TRE-RFP by Draper, we sought to determine how Draper activation might lead to its activation through dAP-1. We screened a large collection of RNAi lines for novel signalling molecules and assayed for roles in glial clearance of degenerating axons. Interestingly, we found that pan-glial expression of UAS-traf $4^{I R}$ (ref. 18) resulted in strong suppression of glial clearance of axonal debris, compared to controls (Fig. 2a,b, Supplementary Fig. 4c,d). TRAF4 is known to act upstream in activation of the JNK signalling pathway through association with the MAP kinase kinase kinase kinase (MAP4K) misshapen (MSN) (ref. 19), which then phosphorylates the downstream MAP3K Slipper and ultimately drives dAP-1 activation of transcriptional targets ${ }^{20}$. A role for TRAF4 in glial engulfment was intriguing given that we previously implicated a number of downstream components of the JNK signalling 
pathway in glial engulfment of axonal debris including dAP-1 (ref. 7), but how this pathway might be activated remained unclear.

Pan glial expression of a dominant negative version of MSN $\left(m s n^{D N}\right)^{21}$ strongly suppressed glial engulfment of axonal debris 5 days after axotomy (Fig. $2 c, d$ ). Consistent with a role for TRAF4 and MSN in activation of the JNK signalling pathway we found that glial expression of traft ${ }^{I R}$ (Fig. 2e,f) or $m s n^{D N}$ (Fig. $\left.2 \mathrm{~g}, \mathrm{~h}, \mathrm{k}\right)$ suppressed activation of the TRE-eGFP reporter. Moreover, we observed that depletion of TRAF4 or expression of MSN ${ }^{\mathrm{DN}}$ suppressed injury-induced increases in Draper that are normally observed 1 day after axonal injury, a
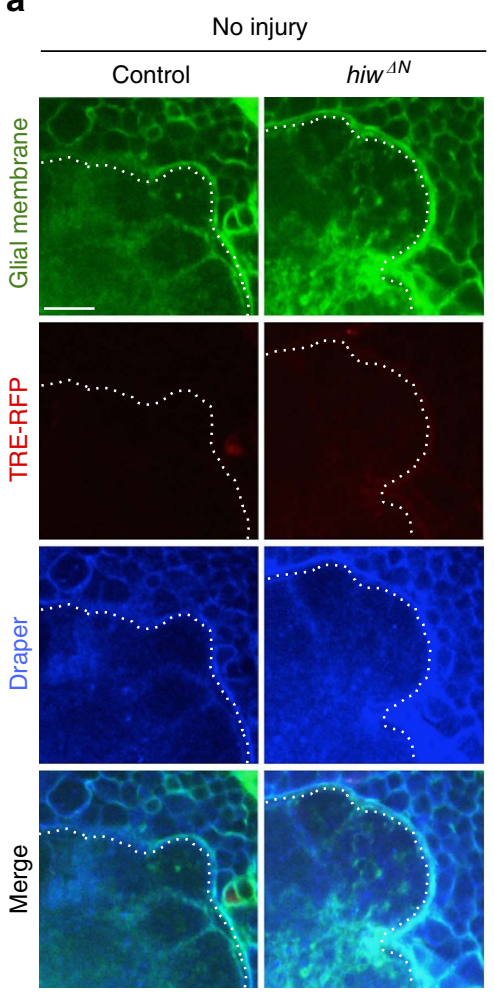

C

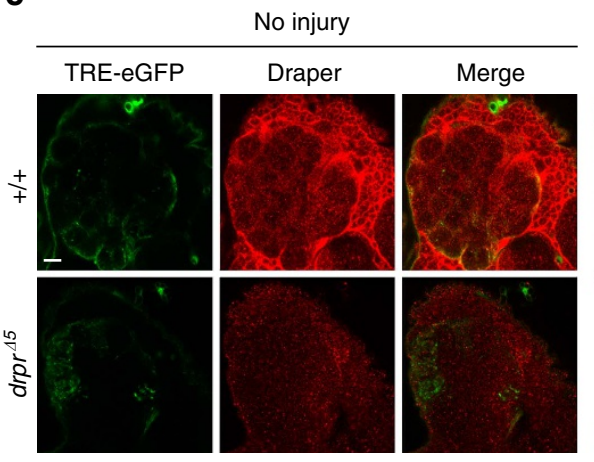

e
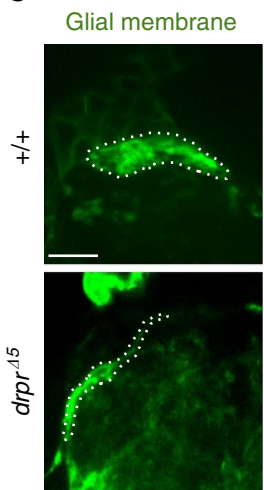

TRE-RFP
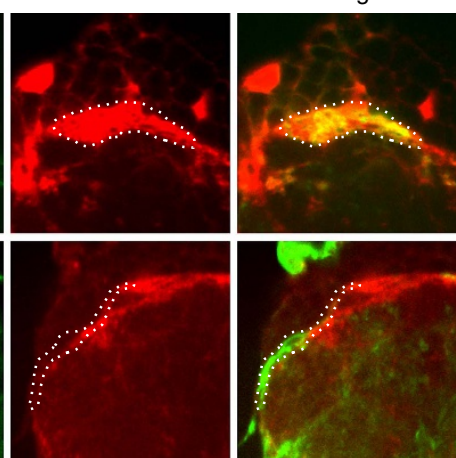

1 dpant
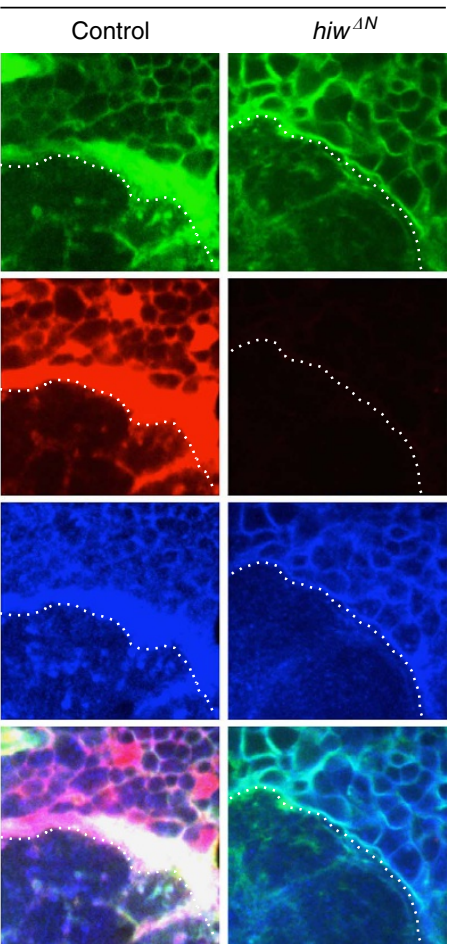

b

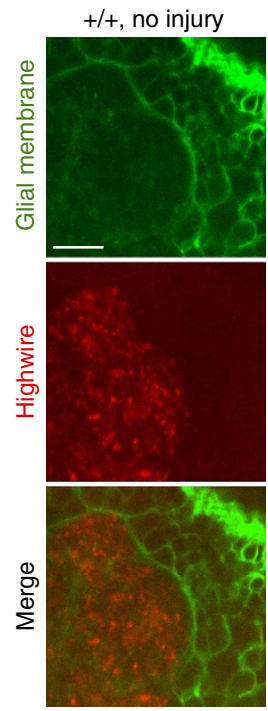

d

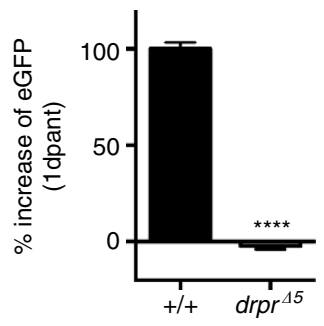


and which we previously reported for downstream components of the JNK signalling pathways. We further noted that in traft ${ }^{I R}$ animals Draper expression was reduced $\sim 50 \%$ even before axon injury (Fig. 2i,j). This phenotype is similar to glial stat $92 e^{R N A i}$ animals, where Draper levels are reduced in uninjured animals, and engulfment defects can be rescued by overexpression of a draper $\mathrm{cDNA}^{6}$. In contrast, we found defects in glial responses to axonal degeneration in the traft ${ }^{I R}$ background did not result from insufficient Draper levels: re-expression of a functional Draper-I transcript (Supplementary Fig. 4a,b) in traft ${ }^{I R}$ animals failed to rescue axonal debris clearance defects (Supplementary Fig. 4c,d). Together these data support a model whereby TRAF4 signals downstream of Draper to promote target engulfment and glial transcriptional reporter activation mediated by STAT92E and dAP-1.

Based on our observation that TRAF4 and MSN regulated injury-induced changes in Draper levels and transcriptional reporters in glia, we sought to define signalling interactions between TRAF4 and MSN, the JNK signalling pathway, and STAT92E. STAT92E activity after axotomy was assayed using the degradable GFP reporter 10xSTAT92E-dGFP (ref. 22), which is transiently activated in glia 1 day after axotomy ${ }^{6}$. In control uninjured animals, GFP expression was absent in brains, but strongly activated in glia surrounding the antennal lobe 1 day after antennal ORN axotomy (Fig. 2l). Glial expression of traf $4^{I R}$ suppressed this increase of GFP expression after axon injury (Fig. 2l,m), indicating that TRAF4 is upstream of the injuryinduced activation of 10xSTAT92E-dGFP. Similarly, blockade of the JNK signalling pathway with basket ${ }^{R N A i}$ (Drosophila JNK), or overexpression of the JNK inhibitor Puckered eliminated the injury-induced upregulation of 10xSTAT92E- $d G F P$ (Fig. 2l,m). Finally, knockdown of Kayak, a component of the dAP-1 transcriptional complex, also led to a significant decrease in 10xSTAT92E- $d$ GFP activation (Fig. $2 \mathrm{l}, \mathrm{m}$ ). Thus STAT92E is genetically downstream of dAP-1 in the context of 10xSTAT92E$d G F P$ reporter activation. Taken together, these genetic data argue that glia activate these transcriptional reporters after axonal injury through a TRAF $4 \rightarrow$ JNK pathway $\rightarrow$ dAP- $1 \rightarrow$ STAT92E signalling cascade that lies downstream of Draper.

TRAF4 binds the Draper intracellular domain. The mechanism by which Draper activates JNK signalling and dAP-1-mediated transcriptional reporter activation has not been defined. The only molecule known to physically associate with Draper to promote downstream signalling events is the Src family kinase Shark, which binds an ITAM in the Draper intracellular domain after phosphorylation by Src42a (ref. 4). We found that depletion of
Shark from all glia by pan-glial expression of UAS-shark ${ }^{R N A i}$ (using the repo-Gal4 driver) blocked activation of TRE-eGFP 1 day after axotomy (Supplementary Fig. 5a,b), and similar RNAi-mediated knockdown of racl or $\operatorname{src} 42 a$ also significantly decreased injury-induced activation of TRE-eGFP (Supplementary Fig. 5c,d). We therefore conclude that dAP-1 transcriptional reporters are genetically downstream of Draper/Shark signalling interactions.

Given that TRAF4 is an adaptor protein, we speculated that TRAF4 might interact with Draper and thereby directly couple Draper to activation of JNK kinase signalling. TRAF4/ MSN have well-defined roles in the activation of Slipper (ref. 20), and in turn the Drosophila JNK signalling pathway. We therefore performed co-immunoprecipitation (co-IP) experiments using Drosophila S2 cell lysates with a Myc-tagged TRAF4 (TRAF4-myc) and HA-tagged Draper I (Draper-I-HA). We found that pull down of Draper-I-HA led to the coprecipitation of TRAF4-myc, indicating that TRAF4 can physically associate with the Draper signalling complex (Fig. 3a). To identify the region through which TRAF4 associates with Draper we assayed the ability of epitope-tagged versions of the N-terminal TRAF4, which is enriched with zinc-finger domains (ZF, aa 1-927) or the C-terminal TRAF domain (TD, aa $928-1458)^{19}$ to associate with Draper. We found that the N-terminal TRAF4 domain co-immunoprecipitated with Draper I-HA, while the C-terminal TRAF domain, which is known to bind MSN (ref. 19), was not detected in the same assay (Fig. 3b,c). We therefore conclude that Draper is bound by the N-terminal domain of TRAF.

To identify the region of Draper-I to which TRAF4 bound, we next made serial deletions in the intracellular domain (Fig. 3d), and performed co-IP with full-length TRAF4-myc. We found that aa 826-894 were essential for Draper-I-TRAF4 interactions (Fig. 3e, $\Delta 826-894$ ), while all other regions were dispensable. We note this includes the evolutionarily conserved NPXY motif that is likely bound by the PTB domain binding protein dCED-6 (ref. 23), which is consistent with the observation that dCED-6 is not required for activation of 10xSTAT92E-dGFP (ref. 6) or JNK signalling (Supplementary Fig. 6) in glia after axotomy. Further analysis of this subdomain revealed that aa 826-854 and 855-594 were both able to bind TRAF4 (Fig. 3d,f), suggesting there may be multiple TRAF4 binding sites in the Draper intracellular domain. The NPXY motif is not absolutely required for TRAF4 binding, as TRAF4 associated strongly with the $\Delta 855-894$ construct lacking this domain (Fig. 3f). Finally, given that both Src42A and Shark are required for activation of TRE-eGFP in response to injury, we assayed whether TRAF4 and Shark binding were reciprocally interdependent. Phosphorylation of Y949 in ITAM, which is

\footnotetext{
Figure 1 | Glial transcriptional activation is downstream of axon degeneration and Draper signalling. (a) Glial responses to axon injury in controls and highwire null $\left(h_{i w}{ }^{\mathrm{N}}\right.$ ) animals after axotomy (1 day after bilateral antennal ablation, 1 dpant). Note glial membrane hypertrophy, activation of the of TRE reporter and upregulation of Draper (anti-Draper) in cohort controls (hiw ${ }^{\Delta N} /+;$ TRE-RFP-16/ + ; repo-Gal4, UAS-mCD8::GFP/ + ). highwire null animals $\left(\right.$ hiw $^{\Delta N} /$ Y; TRE-RFP-16/ + ; repo-Gal4, UAS-mCD8::GFP/ + ), where axon degeneration is blocked, showed no detectable activation of glia. Dotted line delineates edge of the antennal lobe. Representative images were shown from five animals. Scale bar $=10 \mu \mathrm{m}$. (b) Highwire was highly enriched in the synaptic region in the antennal lobe, but not in the surrounding glial cells (labelled with repo-Gal4, UAS-mCD8::GFP/ + ). Representative images were shown from three animals. (c) Glia require Draper to activate TRE reporter 1day after antennal ablation. +/+: TRE-eGFP-16; +/+.drpr ${ }^{45}$ : TRE-eGFP-16; drpr ${ }^{45}$. Representative images were shown from three animals. Scale bar $=10 \mu \mathrm{m}$. (d) Quantification of the increase of eGFP immunoreactivity in (c). $n=15$ each. Unpaired $t$-test, 2 -tailed. ${ }^{\star \star \star \star}: P<0.0001$. (e) Ensheathing glial MARCM clones (green, outlined) responding to axotomy at day 1 post antennal ablation. Control ensheathing glial clones $(+/+)$ became hypertrophic and exhibited strong TRE reporter activation (red) 1 day after antennal ablation, while draper null $\left(\mathrm{drpr}^{45}\right)$ clones did not respond to axotomy (that is lack of membrane hypertrophy, and activation of TRE reporter) while neighbouring control cells were activated. +/+: TRE-RFP-16, UAS-mCD8::GFP/repo-FLP6-2; FRT2A, FRT82B/tub-Gal80, FRT2A, repo-Gal4. drpr ${ }^{45}$ : TRE-RFP-16, UAS-mCD8:: GFP/repo-FLP ${ }^{6-2} ; d r p r^{45}, F R T 2 A / t u b-G a l 80, F R T 2 A$, repo-Gal4. Representative images were shown from four animals. Scale bar $=10 \mu \mathrm{m}$. (f) TRE-RFP reporter activity in cortex glial MARCM clones (dotted line, green) 1 day after antennal ablation. Similar to ensheathing glia, cortex glial clones that lacked Draper $\left(\mathrm{drpr}^{45}\right)$ failed to activate TRE reporter. Genotypes as in (e). Representative images were shown from four animals. Scale bar $=10 \mu \mathrm{m}$.
} 
mediated by Src42A and is bound by Shark (ref. 4), was also dispensable for TRAF4 to associate with Draper (Fig. 3g). Reciprocally, elimination of the TRAF4 interaction domain in Draper I $(\Delta 826-894)$ did not alter Shark association with Draper (Supplementary Fig. 7). Taken together these data indicate that TRAF4 physically associates with the Draper intracellular domain in the region of the NPXY motif, and this interaction occurs in a Shark-independent fashion. These data argue Draper activates the JNK signalling pathway and dAP-1- and STAT92E-dependent transcriptional reporters in a
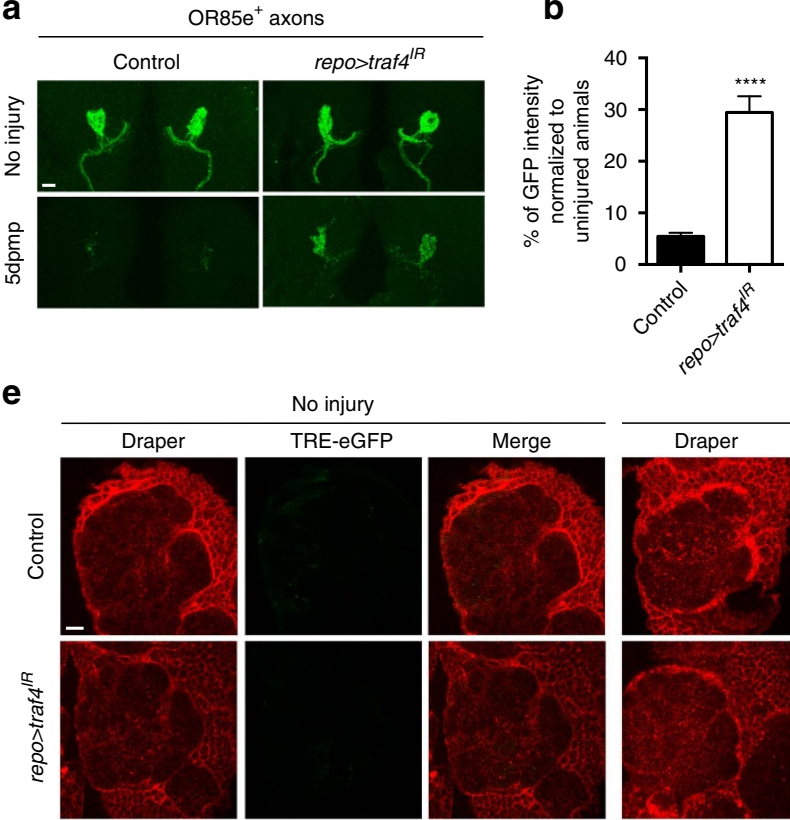

No injury
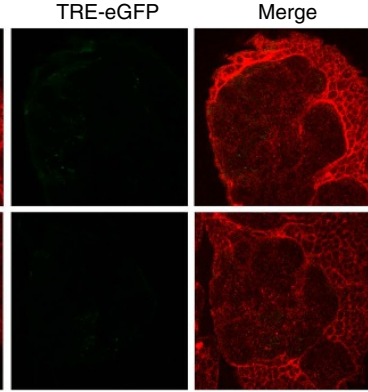

9

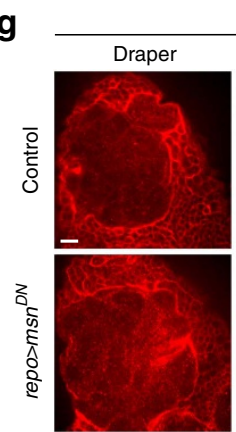

i

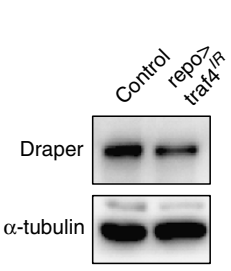

j

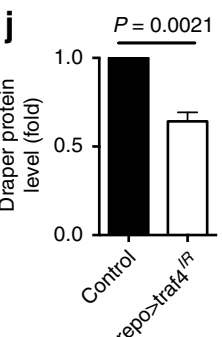

I

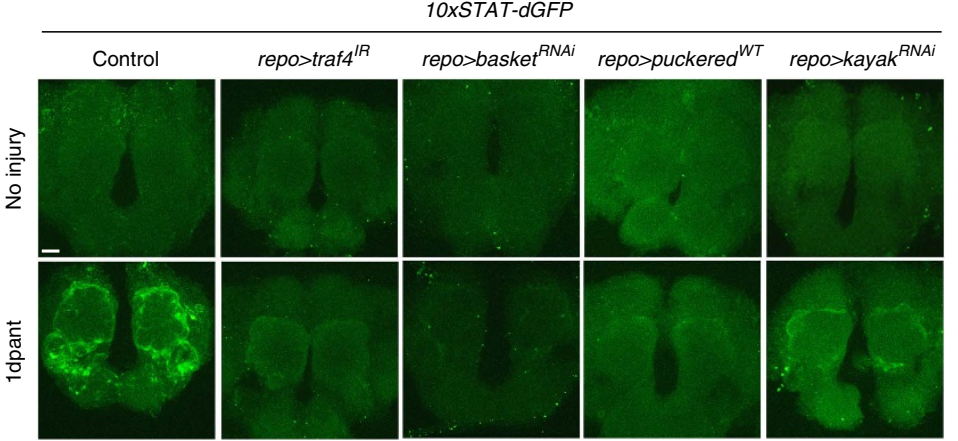

C

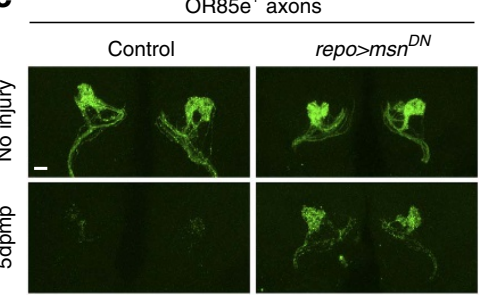

d

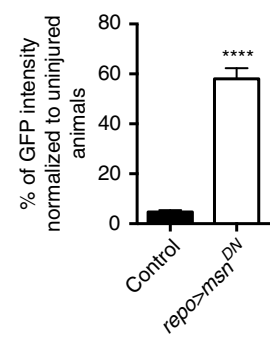

f
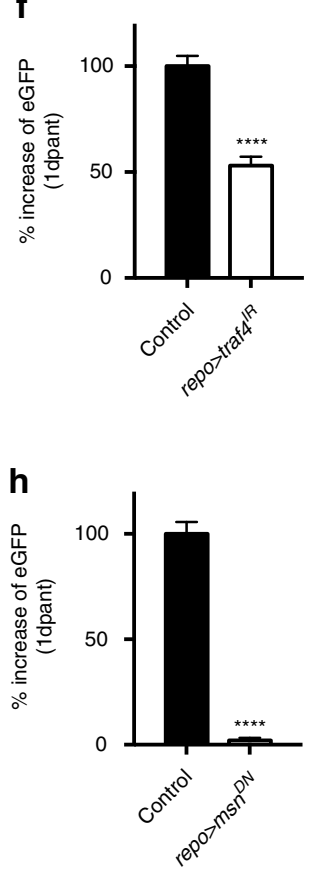

$\mathbf{m}$

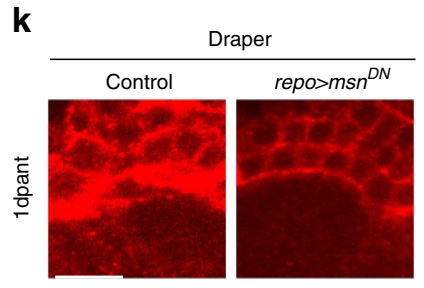

m

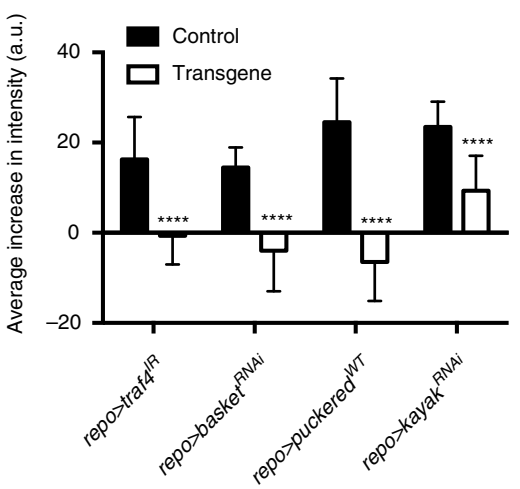


response to axon degeneration by direct association with TRAF4 and activation through MSN.

\section{Discussion}

Activation of JNK/AP-1 and STAT transcriptional activity during reactive glial responses is an evolutionarily conserved feature of Drosophila and mammalian glia ${ }^{24,25}$. However, transmembrane receptors on glia that receive extracellular injury signals and in turn activate AP-1 or STAT-dependent transcriptional changes have not been identified. Our work supports a model whereby the engulfment receptor Draper is activated in response to axonal injury, which in turn promotes JNK signalling through TRAF4 and MSN to alter glial transcription via dAP-1 and STAT92E. Our reliance on well-characterized transcriptional reporters for dAP-1 and STAT92E supports this conclusion, but we note that we have not formally shown transcriptional activation of a downstream target gene, primarily because none have been identified.

Axon degeneration is required to activate glial cells after injury. Blockade of axon degeneration using hiw mutations fully suppresses glial responses to axon injury, including morphological changes and activation of transcriptional reporters, indicating that axonal self-destruction leads to the production of cues that activate glial responses through Draper. Interestingly, based on the activation of glial transcriptional reporters (that is TRE-RFP) even at a distance from the injury site (Fig. 1f), it appears that glia can be activated by degenerating axons even when not in direct contact with severed axons. This observation suggests that axonal 'injury signals' that stimulate Draper signalling may be soluble, or, alternatively that glial-glial signalling may propagate through the glial network in a Draper-dependent manner. Our identification of Draper/TRAF4 interactions as a mechanism for JNK activation raises the intriguing possibility that MEGF10 might play a conserved role in mammalian reactive astrocytes to activate AP-1 or STAT signalling, or other contexts. Indeed, MEGF10 and several other engulfment genes are expressed in astrocytes and MEGF10 is required for developmental synapse pruning ${ }^{26}$.

We propose a simple model where axon self-destruction leads to injury signals that activate Draper, which in turn drives two primary downstream signalling events (Fig. $3 \mathrm{~h}$ ). In the first, Draper promotes cytoskeletal rearrangements essential for phagocytic activity through Src42a, Shark and Traf4 in direct association with Draper, and downstream GEF complexes such as Crk-II/Mbc/dCed-12 and DRK/DOS/SOS (refs 5,27). These signalling pathways mediate membrane extension to, and internalization of engulfment targets. At the same time we propose that Draper activation leads to glial transcriptional responses to injury, including upregulation of dAP-1 and STAT92E transcriptional targets (ref. 12), although these need to be identified and validated. Consistent with the draper gene being a primary transcriptional target of this cascade, re-expression of Draper in glia is sufficient to rescue engulfment defects after knockdown of JNK, dAP-1 and STAT92E (refs 6,7). However, re-expression of Draper is not sufficient to rescue engulfment defects in animals lacking glial Src42a or TRAF4 (Supplementary Fig. 4). The simplest interpretation of these data is that Draper, TRAF4 and Shark function upstream in the signalling pathway and are required for both internalization of targets and activation of transcriptional responses. This model is supported by our finding that TRAF4 and Shark directly bind Draper. Depletion of TRAF4 or STAT92E from glia alters basal levels of Draper, while, surprisingly, RNAi mediates suppression of dJNK or dAP-1 does not. This may indicate additional signalling pathways promote cross-talk between TRAF4 and STAT92e during transcriptional regulation of basal levels of draper, which is independent of dJNK.

Draper/MEGF10-mediated signalling through TRAF4 may also be engaged during developmental synapse elimination or reactive gliosis in mammals, but this awaits further exploration. Draper/MEGF10 has recently been shown to have potentially non-phagocytic signalling roles including activation of autophagy in Drosophila salivary glands ${ }^{28}$ and the tiling of starburst amacrine cells ref. 17. Exploring the roles for TRAF4, dAP-1 and STAT could provide further important molecular insight into Draper/MEGF10 regulation of transcription changes in these physiological contexts.

\section{Methods}

Fly strains. Drosophila melanogaster strains used in this study include: OR85e-mCD8::GFP/CyO (ref. 29), repo-Gal4/TM3 (ref. 30), nSyb-Gal4, $5 x U A S-m C D 8:: G F P$ (ref. 31), UAS-traf4 ${ }^{I R}$ (gift from T. Xu, Yale U) ${ }^{18}$, UAS-drprI(24127)-HA, TRE-eGFP-16 (ref. 13), TRE-RFP(dsRed.T4)-16 (ref. 13), tub-Gal80 ${ }^{\text {ts }}$, OR85e-mCD8::GFP/CyO (ref. 27), UAS-msn ${ }^{D N}$ (gift from M. Leptin, Cologne U, Cologne) ${ }^{32}$, 10xSTAT92E-GFP; repo-Gal4/TM3 (ref. 6),

10xSTAT92E-dGFP; repo-Gal4/TM3 (ref. 6), UAS-puckered (Bloomington Stock Center), $d r p r^{45 r e c 9}$ (ref. 33), repo-FLP ${ }^{6-2}$ (II) ${ }^{34}, F R T 2 A$, FRT82B (III) ${ }^{33}$, repo-Gal4, UAS-mCD8::GFP/TM3 (ref. 6), hiw ${ }^{\Delta N}$ (ref. 35), UAS-gfp::hiw (ref. 31),

tub-Gal80 ${ }^{\text {ts }}$; repo-Gal4, UAS-mCD8::GFP/TM3 (ref. 27), UAS-Rac1 ${ }^{\text {N17 }}$ (ref. 36) and UAS-shark ${ }^{R N A i \# 6 b}$ (ref. 4). The following Drosophila strains were generated during the study according to standard procedure: TRE-eGFP-16, tub-Gal80 ${ }^{\text {ts }}$ (II), $d r p r^{45 r e c 9}$, FRT2A/TM6, TRE-RFP-16, UAS-mCD8::GFP (II), tub-Gal80, FRT2A, repo-Gal4/TM3, UAS-drprI-HA, drpr ${ }^{45 r e c 9} /$ TM6, repo-Gal4, drpr ${ }^{45 r e c 9} /$ TM6

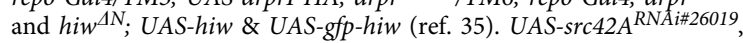

\footnotetext{
Figure 2 | TRAF4 and MSN are upstream of JNK- and STAT92E-mediated responses to axotomy. (a) RNAi-mediated depletion of TRAF4 in glia (repo > traf4 ${ }^{I R}$ : OR85e-mCD8::GFP/UAS-traf4 ${ }^{I R}$; repo-Gal4/+) resulted in a delay in axonal debris clearance 5 days after maxillary malp ablation ( 5 dpmp) compared to controls (OR85e-mCD8::GFP/ + ; repo-Gal4/+). OR85e $\mathrm{e}^{+}$maxillary nerves were labelled with $\mathrm{mCD} 8:: \mathrm{GFP}$. Scale bar for all $=10 \mu \mathrm{m}$. (b) Quantification from (a). $n=10$. Unpaired t-test, 2-tailed (for all). (c) Expression of dominant-negative MSN ( $\mathrm{msn}^{D N}$ ) suppressed glial clearance of axonal debris. Control: OR85e-mCD8::GFP, tub-Gal80ts/ + ; repo-Gal4/+. Repo >msn DN: OR85e-mCD8::GFP, tub-Gal80 ts /UAS-msn DN; repo-Gal4/+. Representative images were shown (control: $n=10$; repo $>m s n^{D N}: n=9$ ). (d) Quantification from (c). (e) TRE reporter activity was suppressed in TRAF4 RNAi animals (repo $>$ traf4 $\left.{ }^{I R}\right) 1$ day after antennal ablation. Control: TRE-eGFP-16/+; repo-Gal4/ + . Repo > traf4 ${ }^{I R}$ : TRE-eGFP-16/UAS-traf4IR; repo-Gal4/+. (f) Quantification of the eGFP immunoreactivity in (e). $n=25$ each. (g) TRE reporter activity was inhibited 1 day after antennal ablation by glial expression of MSNDN. Control: TRE-eGFP-16, tub-Gal80ts/ +; repo-Gal4/+. Repo>msn DN: TRE-eGFP-16, tub-Gal80ts/UAS-msn DN; repo-Gal4/ +. (h) Quantification from $(\mathbf{g}) . n=15$ each. (i) Draper expression was reduced on western blots when TRAF4 was knocked down in glia from adult dissected central brains. $\alpha$-tubulin was used as loading control. Representative images were shown from three repeats. Control: repo-Gal4/ + . Repo $>$ traf4 ${ }^{I R}:$ UAS-traf 4 IR $/+$; repo-Gal4/+. (j) Quantification of (i). $n=3$. Unpaired t-test, 2-tailed. (k) Higher magnification images of dashed squares in ( $\mathbf{g}$ ) showed the lack of Draper increase 1 day after antennal ablation in $\mathrm{msn}^{\mathrm{DN}}$ animals. (I) STAT92E-mediated dGFP (degradable GFP) expression was induced in glia 1 day after antennal ablation in control animals (10xSTAT92E-dGFP/ + ; repo-Gal4/ + ), but blocked in animals where JNK signalling cascade was interrupted by glial

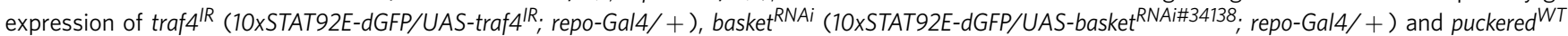

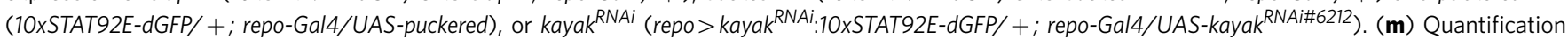
of the increase of dGFP immunoreactivity in (I). Repo $>\operatorname{traf}^{I R}: n=15$ each. Repo $>$ basket $^{R N A i}$, repo $>$ puckered ${ }^{W T}$ and repo $>$ kayak ${ }^{R N A i}: n=25$ each. Unpaired t-test for each group, 2-tailed.
} 


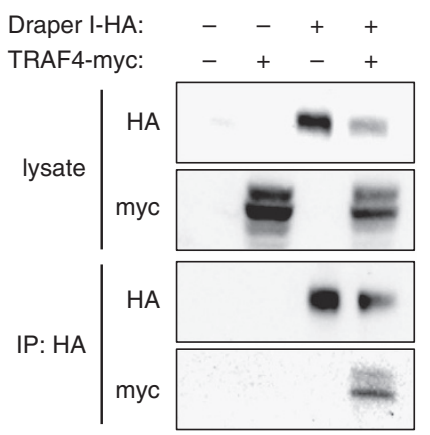

b

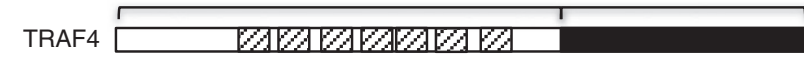

Q : zinc-finger domain

: TRAF domain

C

Draper

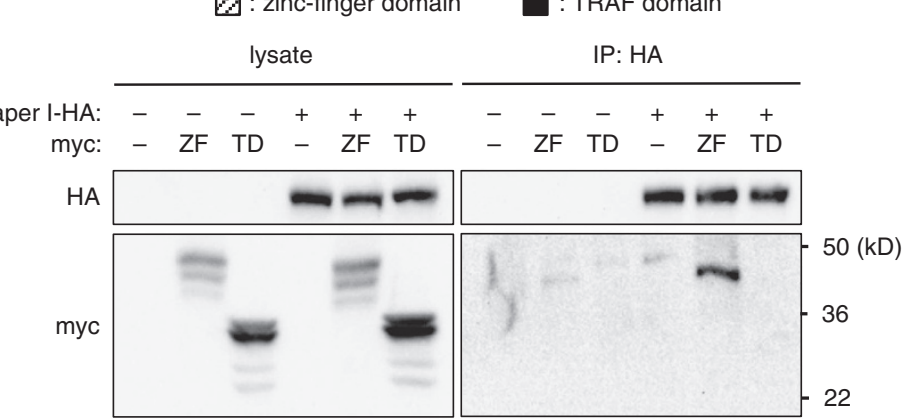

d

: transmembrane domain $\quad \mathbb{Q}:$ NPXY $\quad \mathbf{Q}:$ ITAM

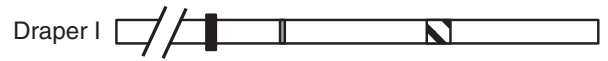

8826-894 प $\square$ Q

8855-933 प

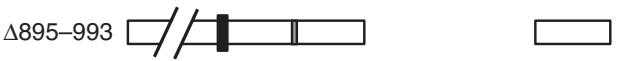

1953-1011

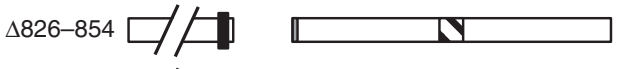

855-894 प

$\mathbf{N}$

f
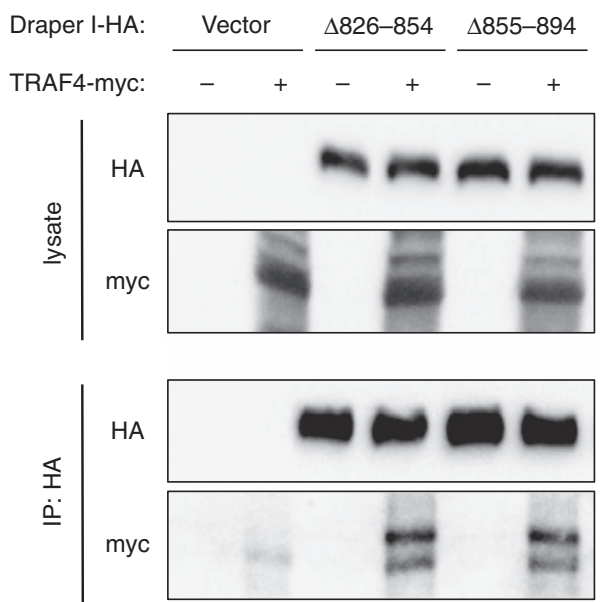

e
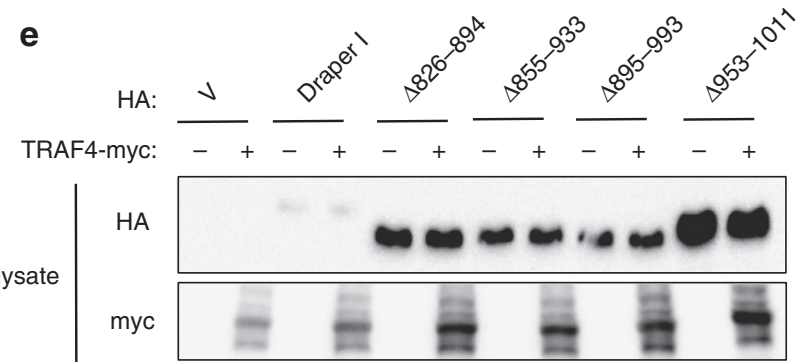

IP: HA

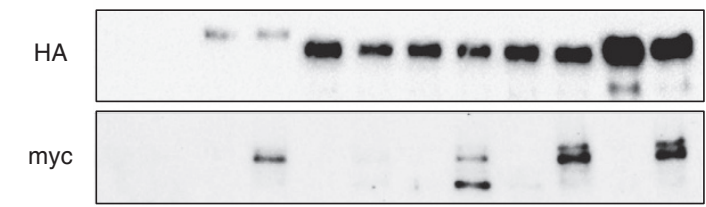

g

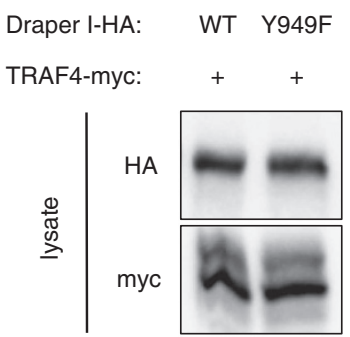

h

Axon degeneration

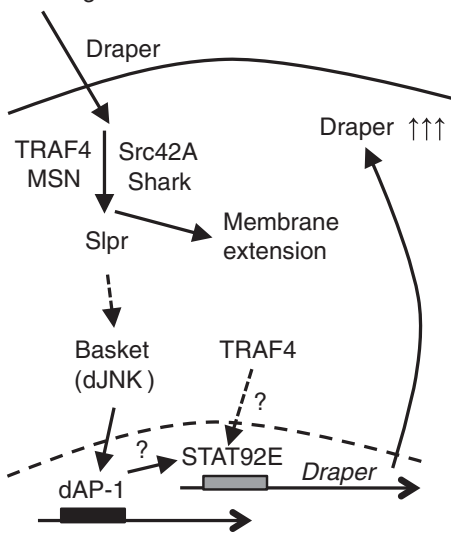

Figure 3 | TRAF4 physically associates with Draper independently of Shark. (a) Western blots of co-immunoprecipitations from Drosophila S2 cells showed TRAF4-myc associated with Draper I-HA. All the blots shown are representative images after three repeats. (b) Schematic drawing of Drosophila TRAF4. ZF: zinc-finger enriched domain. TD: TRAF domain. (c) Draper I-HA interacted with the ZF region of TRAF4 but not the TRAF domain.

(d) Schematic drawing of deletions in the Draper I intracellular for different constructs. The extracellular domain $(/ /)$ was not drawn to scale. All the Draper I constructs were C-terminally tagged with HA. (e) The deletion of a.a. 826-894 decreased the association between Draper I and TRAF4. V: vector alone. (f) Draper I $\Delta 826-854$ and $\Delta 855-894$, bound TRAF4-myc at similar levels, suggesting these two regions are equally important in mediating the interaction between TRAF4 and Draper I. (g) The mutation Y949F in Draper, which is required for Shark to bind Draper I, did not affect the interaction between Draper I and TRAF4, indicating that the interaction between TRAF4 and Draper I does not require Shark association. (h) Proposed model of Draper-mediated glial transcriptional responses to axon degeneration. Draper is cell-autonomously required for glia to respond to axon degeneration and genetically upstream of JNK signalling cascade activation. The two binding partners of Draper, TRAF4 and Shark, are also upstream of JNK signalling cascade, likely through the activation of Slpr. We propose activation of JNK signalling cascade induces dAP-1-mediated gene transcription, which is also upstream of STAT92E-dependent transcriptional activation. The activation of STAT92E leads to the upregulation of Draper and facilitation of glial engulfment of axonal debris. 
UAS-basket ${ }^{R N A i \# 34138}$ and UAS-kayak ${ }^{R N A i \# 6212}$ were obtained from Vienna Drosophila Resource Center.

Antibodies. For primary antibodies used in this study: Mouse anti-GFP clone 3E6 (Molecular Probes A-11120 1:200 for IFA; 1:2000 for WB), rabbit anti-Draper (ref. 37) (1:500 for IFA and 1:1000 for WB after pre-absorbed with $y w$ embryos overnight), mouse anti- $\alpha$-tubulin (DM1A) (Sigma T9026, 1:1000), rat anti-mCherry (Invitrogen M11217, 1:1000), rat anti-HA (3F10) (Roche 1-867-423, 1:2000 for WB), mouse anti-myc (9E10) (Millipore 05-419, 1:5000), mouse anti-Hiw (6H4) (Developmental Studies Hybridoma Bank, 1:1000) and mouse anti-Repo (Developmental Studies Hybridoma Bank, 1:5). FITC anti-mouse IgG (715-095-150), Cy3 anti-rabbit IgG (711-165-152), Cy3-anti-rat IgG (712-1165-150), Cy3 anti-mouse IgG (715-165-150) and Cy5 anti-rabbit IgG antibodies (711-165-152) were from Jackson ImmunoResearch Laboratories (all 1:100), as well as horseradish peroxidase-conjugated anti-rat antibodies (712-035-150, 1:6000). Both horseradish peroxidase-conjugated anti-mouse (ab6808), and anti-rabbit IgG (ab6721-1) were from Abcam (1:6000).

Injury protocol and adult brain dissection. Drosophila cultures were kept at $25^{\circ} \mathrm{C}$ except for the experiments involving UAS- $m s n^{D N}$ and UAS-src $42 A^{R N A i \# 26019}$, due to the lethality they caused when overexpressed using repo-Gal4. Temperaturesensitive GAL80 $\left(\text { Gal } 80^{t 5}\right)^{38}$ was used to inhibit the activity of GAL4 at $18^{\circ} \mathrm{C}$ before fly eclosion and then deactivated at $29^{\circ} \mathrm{C}$ for at least 5 days before injury. Flies continued to grow at $29^{\circ} \mathrm{C}$ until the day of dissection. Maxillary palp and the third antennal segment ablation were performed using a pair of fine forceps under dissection microscope. For immunocytochemistry, heads were first fixed and permeabilized in $4 \%$ formaldehyde/ $0.1 \%$ Triton X-100/PBS for $20 \mathrm{~min}$ at room temperature (R.T.). After washed with PBST (0.1\% Triton X-100/PBS) for five times, brains were dissected in PBST and post-fixed with $4 \%$ formaldehyde/ $0.1 \%$ Triton X-100/PBS for another $15 \mathrm{~min}$. Fixed brains were then washed for five times with PBST, blocked in $0.1 \%$ Triton X-100/5\% BSA/PBS for 30 min at R.T., and incubated with primary antibodies in PBST at $4{ }^{\circ} \mathrm{C}$ overnight or two nights for anti-Draper antibody. The next day, the brains were washed five times with PBST and then incubated with secondary antibodies in PBST for $2 \mathrm{~h}$ at R.T. or $4 \mathrm{~h}$ for Draper staining. After washed with PBST for five times, the brains were then mounted with VECTASHIELD Mounting Medium (Vector Labs) and stored at $4{ }^{\circ} \mathrm{C}$ before imaging within 2 weeks. For western blot analysis, heads from adult flies aged at $25^{\circ} \mathrm{C}$ for at least 5 days after eclosion were isolated and immediately dissected in chilled PBS. Only the central brains (without the optic lobes) were collected and prepared for western blot analysis. Equal number of males and females were used for each experiment. Dissected brains were spinned down at the highest speed for $1 \mathrm{~min}$ and then lysed in 1X Laemmli lysis buffer ( $2 \mu \mathrm{l}$ per brain) on ice for $20 \mathrm{~min}$. The mixture was ground for $30 \mathrm{~s}$ on ice and centrifuged at the highest speed for $10 \mathrm{~min}$ at $4{ }^{\circ} \mathrm{C}$. Only the supernatants were collected for SDS-PAGE analysis and western blotting. Images have been cropped for presentation in Fig. 2i. Full-size images are presented in Supplementary Fig. 8.

\section{Confocal microcopy and image analysis. Confocal microscopy settings were} always kept constant throughout the same set of experiments. Zeiss LSM5 Pascal confocal microscope was used for all axonal debris clearance, TRE-eGFP reporter (except for $U A S-m s n^{D N}$ ), and STAT92E reporter assays with Zeiss Plan-Apochromat $\times 63 / 1.4 \mathrm{NA}$ oil objective lens. Glial MARCM clones, glial activation in hiw $w^{\Delta N}$ animals and TRE-eGFP reporter activity in $m s n^{D N}$ animals were analysed using Zeiss Axio Imager.M2 spinning-disk confocal microscope with Zeiss C-Apochromat $\times 40 / 1.2 \mathrm{NA}$ water objective lens. For quantification of the fluorescence intensity, the centre single $z$-section was identified from each image stack of the relevance glomerulus or central adult brain, and pixel intensity was measured using ImageJ (National Institute of Health).

DNA cloning and Draper I intracellular domain deletions. To clone TRAF4 cDNA, forward primer containing KpnI and reverse primer containing SacII restriction site (all the primer sequences are listed in Supplementary Table 1) were used to amplify TRAF4 cDNA from BDGP (Berkeley Drosophila Genome Project) cDNA clone LD20987. An approximate $1.6 \mathrm{~kb}$ cDNA was amplified by Phusion High-fidelity DNA polymerase (New England BioLabs), purified with QIAquick PCR Purification (QIAGEN) and then digested by KpnI and SacII. Digested DNA fragment was ligated to pUAST-CT-myc vector that has $6 \mathrm{x}$ myc sequence (MEQKLISEEDLNE) at the C-terminus, using T4 DNA ligase (New England BioLabs). The N-terminal (ZF, a.a. 1-309) and the C-terminal TRAF domain (TD, a.a. 310-494) of TRAF4 were isolated from pUAST-TRAF4-myc using the primers listed in Supplementary Table 1. Amplified PCR products were digested with both BgIII and SacII, respectively, and then ligated to pUAST-CT-myc vector. Internal deletions in the Draper I intracellular domain were generated using GeneArt Seamless Cloning and Assembly Enzyme Mix (Life Technologies). See Supplementary Table 1 for the exact sequences of the primers used to amplify Draper I fragments from pAc5-DraperI-HA. pAc5 vector was linearized by KpnI and XbaI, purified with QIAquick PCR Purification (QIAGEN), and then incubated with purified Draper I-HA fragments in $1 \mathrm{x}$ seamless enzyme mix at R.T. for $15 \mathrm{~min}$ followed by standard bacterial transformation procedure. To make C-terminally truncated Draper I $(\Delta 953-1101)$, the forward primer (containing a KpnI restriction site and the sequences matching Draper I nucleotide (nt.) 1-16), and the reverse primer (containing a XbaI restriction site, a stop codon (TAG), 1x HA DNA sequence and Draper I nt. 2838-2856) were used to amplify Draper I cDNA from pAc5-Draper I. PCR product was purified and digested with $\mathrm{KpnI}$ and $\mathrm{XbaI}$, and then ligated to pAc5 vector. All the restrict enzymes used were purchased from New England BioLabs.

Schneider 2 cell culture and co-immunoprecipitation. Drosophila S2 cells were cultured and maintained in 10\% HyClone Fetal Bovine Serine/SFX-Insect Media (Fisher Scientific) with $1 \%$ penicillin-streptomycin solution (Sigma-Aldrich) at $25^{\circ} \mathrm{C}$. For immunoprecipitation of Draper I-HA, $2 \times 10^{6} \mathrm{~S} 2$ cells were seeded per well in a six-well plate the day before DNA transfection, where $100 \mathrm{ng}$ of each appropriate DNA vector were mixed and incubated with Effectene Transfection Reagents (QIAGEN), as described in manufacturer's instruction. Two days after transfection, cells were lysed in our modified NP-40 lysis buffer ( $1 \% \mathrm{NP}-40,10 \mathrm{mM}$ Tris- $\mathrm{HCl} \mathrm{pH} 7.5,50 \mathrm{mM} \mathrm{NaCl}, 30 \mathrm{mM} \mathrm{Na}{ }_{4} \mathrm{P}_{2} \mathrm{O}_{7}, 50 \mathrm{mM} \mathrm{NaF}, 100 \mu \mathrm{M} \mathrm{Na}_{3} \mathrm{VO}_{4}$, $5 \mu \mathrm{M} \mathrm{ZnCl} 2$ and protease inhibitor cocktail purchased from Roche $)^{12}$. Rat anti-HA monoclonal antibody ( $0.3 \mu \mathrm{g}$ per sample) was incubated with Protein $\mathrm{G}$ beads (Sigma-Aldrich) at $4{ }^{\circ} \mathrm{C}$ for $3 \mathrm{~h}$ before incubated with pre-cleared cell lysates at $4^{\circ} \mathrm{C}$ overnight. After washed at least eight times to reduce non-specific binding, proteins on beads were eluted in SDS loading buffer $(60 \mathrm{mM}$ Tris $\mathrm{pH} 6.8$, $10 \%$ glycerol, $2 \%$ SDS, $1 \% \beta$-mercaptoethanol and $0.01 \%$ bromophenol blue) and boiled at $100^{\circ} \mathrm{C}$ for $5 \mathrm{~min}$, following a 2 -min centrifugation at $16,000 \mathrm{~g}$. Supernatants were loaded into $4-15 \%$ gradient Tris-HCl ReadyGel (Bio-Rad). SeeBlue Pre-stained Protein Standard (Life Technologies) was used (10 $\mu$ l per well) to determine protein molecular weight. Samples were transferred to nitrocellulose membrane (Bio-Rad) after electrophoresis. Before incubated with corresponding primary antibodies at $4{ }^{\circ} \mathrm{C}$ overnight, membrane was blocked in $5 \%$ milk $/ 0.01 \%$ Tween-20/PBS at R.T. for $1 \mathrm{~h}$. Membrane was then washed with $0.01 \%$ Tween-20/ PBS two times in $30 \mathrm{~min}$ and then probed with corresponding secondary antibodies at R.T. for $1 \mathrm{~h}$, followed by another two washes in $30 \mathrm{~min}$. Immunoreactivity was detected using Clarity Western ECL Substrate (Bio-Rad) or ECL Prime Western Blotting Detection Reagent (Amersham) for TRAF4-myc after co-IP. Image acquisition and protein quantification were conducted using ChemiDoc ${ }^{\mathrm{TM}} \mathrm{MP}$ system (Bio-Rad) and signal saturation was avoided during acquisition. Images have been cropped for presentation in Fig. 3a,c,e-g. Full-size images are presented in Supplementary Fig. 8. All results showed were representative blots after at least three repeats.

Statistical analysis. All error bars represent s.e.m. Unpaired Student's $t$-test (2-tailed), one-way ANOVA, and post-hoc analyses were carried out using GraphPad Prism 6 (GraphPad Software). Data distribution was assumed to be normal, although this was not formally tested. No statistical methods were used to predetermine sample sizes, but our sample sizes are similar to those reported in previous publications. Also, data collection and analysis were not performed blind owing to the conditions of the experiments. Data were not collected and processed randomly. Animals were assigned to the various experimental groups on the basis of genotype.

Data availability. The authors declare that the experimental results supporting the findings are included in the article and the Supplementary Information or are available upon request.

\section{References}

1. Zhou, Z., Hartwieg, E. \& Horvitz, H. R. CED-1 is a transmembrane receptor that mediates cell corpse engulfment in C. elegans. Cell 104, 43-56 (2001).

2. MacDonald, J. M. et al. The Drosophila cell corpse engulfment receptor Draper mediates glial clearance of severed axons. Neuron 50, 869-881 (2006).

3. Scheib, J. L., Sullivan, C. S. \& Carter, B. D. Jedi-1 and MEGF10 signal engulfment of apoptotic neurons through the tyrosine kinase Syk. J. Neurosci. 32, 13022-13031 (2012).

4. Ziegenfuss, J. S. et al. Draper-dependent glial phagocytic activity is mediated by Src and Syk family kinase signalling. Nature 453, 935-939 (2008).

5. Ziegenfuss, J. S., Doherty, J. \& Freeman, M. R. Distinct molecular pathways mediate glial activation and engulfment of axonal debris after axotomy. Nat. Neurosci. 15, 979-987 (2012).

6. Doherty, J. et al. PI3K signaling and Stat92E converge to modulate glial responsiveness to axonal injury. PLoS Biol. 12, e1001985 (2014).

7. MacDonald, J. M., Doherty, J., Hackett, R. \& Freeman, M. R. The c-Jun kinase signaling cascade promotes glial engulfment activity through activation of draper and phagocytic function. Cell Death Differ. 20, 1140-1148 (2013).

8. Burda, J. E. \& Sofroniew, M. V. Reactive gliosis and the multicellular response to CNS damage and disease. Neuron 81, 229-248 (2014).

9. Middeldorp, J. \& Hol, E. M. GFAP in health and disease. Prog. Neurobiol. 93, 421-443 (2011).

10. Persson, L. et al. S-100 protein and neuron-specific enolase in cerebrospinal fluid and serum: markers of cell damage in human central nervous system. Stroke 18, 911-918 (1981). 
11. Arthur-Farraj, P. J. et al. c-Jun reprograms Schwann cells of injured nerves to generate a repair cell essential for regeneration. Neuron 75, 633-647 (2012).

12. Logan, M. A. et al. Negative regulation of glial engulfment activity by Draper terminates glial responses to axon injury. Nat. Neurosci. 15, 722-730 (2012).

13. Chatterjee, N. \& Bohmann, D. A Versatile ФC31 based reporter system for measuring AP-1 and Nrf2 signaling in Drosophila and in tissue culture. PLoS ONE 7, e34063 (2012).

14. Xiong, X. et al. The Highwire ubiquitin ligase promotes axonal degeneration by tuning levels of Nmnat protein. PLoS Biol. 10, e1001440 (2012).

15. Lee, T. \& Luo, L. Mosaic analysis with a repressible cell marker for studies of gene function in neuronal morphogenesis. Neuron 22, 451-461 (1999).

16. Doherty, J., Logan, M. A., Taşdemir, O. E. \& Freeman, M. R. Ensheathing glia function as phagocytes in the adult Drosophila brain. J. Neurosci. 29, 4768-4781 (2009).

17. Kay, J. N., Chu, M. W. \& Sanes, J. R. MEGF10 and MEGF11 mediate homotypic interactions required for mosaic spacing of retinal neurons. Nature 483, 465-469 (2012).

18. Igaki, T., Pagliarini, R. A. \& Xu, T. Loss of cell polarity drives tumor growth and invasion through JNK activation in Drosophila. Curr. Biol. 16, 1139-1146 (2006).

19. Liu, H., Su, Y.-C., Becker, E., Treisman, J. \& Skolnik, E. Y. A Drosophila TNF-receptor-associated factor (TRAF) binds the Ste20 kinase Misshapen and activates Jun kinase. Curr. Biol. 9, 101-104 (1999).

20. Garlena, R. A., Gonda, R. L., Green, A. B., Pileggi, R. M. \& Stronach, B. Regulation of mixed-lineage kinase activation in JNK-dependent morphogenesis. J. Cell Sci. 123, 3177-3188 (2010).

21. Houalla, T., Hien Vuong, D., Ruan, W., Suter, B. \& Rao, Y. The Ste20-like kinase misshapen functions together with Bicaudal-D and dynein in driving nuclear migration in the developing Drosophila eye. Mech. Dev. 122, 97-108 (2005).

22. Bach, E. A. et al. GFP reporters detect the activation of the Drosophila JAK/STAT pathway in vivo. Gene Expr. Patterns 7, 323-331 (2007).

23. Su, H. P. et al. Interaction of CED-6/GULP, an adapter protein involved in engulfment of apoptotic cells with CED-1 and CD91/low density lipoprotein receptor-related protein (LRP). J. Biol. Chem. 277, 11772-11779 (2002).

24. Gadea, A., Schinelli, S. \& Gallo, V. Endothelin-1 regulates astrocyte proliferation and reactive gliosis via a JNK/c-Jun signaling pathway. J. Neurosci. 28, 2394-2408 (2008).

25. Oliva, A. A., Kang, Y., Sanchez-Molano, J., Furones, C. \& Atkins, C. M. STAT3 signaling after traumatic brain injury. J. Neurochem. 120, 710-720 (2012).

26. Chung, W.-S. et al. Astrocytes mediate synapse elimination through MEGF10 and MERTK pathways. Nature 504, 394-400 (2013).

27. Lu, T.-Y., Doherty, J. \& Freeman, M. R. DRK/DOS/SOS converge with $\mathrm{Crk} / \mathrm{Mbc} / \mathrm{dCed}-12$ to activate Rac1 during glial engulfment of axonal debris. Proc. Natl Acad. Sci. USA 111, 12544-12549 (2014).

28. McPhee, C. K., Logan, M. A., Freeman, M. R. \& Baehrecke, E. H. Activation of autophagy during cell death requires the engulfment receptor Draper. Nature 465, 1093-1096 (2010).

29. Couto, A., Alenius, M. \& Dickson, B. J. Molecular, anatomical, and functional organization of the Drosophila olfactory system. Curr. Biol. 15, 1535-1547 (2005).

30. Sepp, K. J., Schulte, J. \& Auld, V. J. Peripheral glia direct axon guidance across the CNS/PNS transition zone. Dev. Biol. 238, 47-63 (2001).

31. Nagarkar-Jaiswal, S. et al. A library of MiMICs allows tagging of genes and reversible, spatial and temporal knockdown of proteins in Drosophila. Elife 4, 2743-2758 (2015)
32. Mathew, S. J., Rembold, M. \& Leptin, M. Role for Traf4 in polarizing adheren junctions as a prerequisite for efficient cell shape changes. Mol. Cell Biol. 31, 4978-4993 (2011).

33. Neukomm, L. J., Burdett, T. C., Gonzalez, M. A., Züchner, S. \& Freeman, M. R Rapid in vivo forward genetic approach for identifying axon death genes in Drosophila. Proc. Natl Acad. Sci. USA 111, 9965-9970 (2014).

34. Stork, T., Sheehan, A., Tasdemir-Yilmaz, O. E. \& Freeman, M. R. Neuron-glia interactions through the heartless FGF receptor signaling pathway mediate morphogenesis of Drosophila astrocytes. Neuron 83, 388-403 (2014).

35. Wu, C., Wairkar, Y. P., Collins, C. A. \& DiAntonio, A. Highwire function at the Drosophila neuromuscular junction: spatial, structural, and temporal requirements. J. Neurosci. 25, 9557-9566 (2005).

36. Luo, L., Liao, Y. J., Jan, L. Y. \& Jan, Y. N. Distinct morphogenetic functions of similar small GTPases: Drosophila Dracl is involved in axonal outgrowth and myoblast fusion. Genes Dev. 8, 1787-1802 (1994).

37. Freeman, M. R., Delrow, J., Kim, J., Johnson, E. \& Doe, C. Q. Unwrapping glial biology: $\mathrm{Gcm}$ target genes regulating glial development, diversification, and function. Neuron 38, 567-580 (2003).

38. McGuire, S. E., Mao, Z. \& Davis, R. L. Spatiotemporal gene expression targeting with the TARGET and gene-switch systems in Drosophila. Sci. STKE 2004, pl6 (2004).

\section{Acknowledgements}

We thank Tian Xu (Yale U) and Maria Leptin (Cologne U) for providing fly strains, and Jaeda Coutinho-Budd for critical reading of the manuscript. This work was supported by NIH RO1 NS053538 to M.R.F., and during the period of this study M.R.F. was an Investigator with the Howard Hughes Medical Institute.

\section{Author contributions}

T.-Y.L., J.M.M. and M.R.F. designed research. All the experiments were performed by T.-Y.L, except the re-expression of Highwire in the neurons of Highwire null animals (performed by L.J.N.). A.E.S. cloned full-length TRAF4 cDNA. R.B. assisted with cell culture and co-IP. M.A.L. provided transgenic flies.

\section{Additional information}

Supplementary Information accompanies this paper at http://www.nature.com/ naturecommunications

Competing financial interests: The authors declare no competing financial interests.

Reprints and permission information is available online at http://npg.nature.com/ reprintsandpermissions/

How to cite this article: Lu, T.-Y. et al. Axon degeneration induces glial responses through Draper-TRAF4-JNK signalling. Nat. Commun. 8, 14355 doi: $10.1038 /$ ncomms14355 (2017).

Publisher's note: Springer Nature remains neutral with regard to jurisdictional claims in published maps and institutional affiliations.

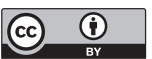

This work is licensed under a Creative Commons Attribution 4.0 International License. The images or other third party material in this article are included in the article's Creative Commons license, unless indicated otherwise in the credit line; if the material is not included under the Creative Commons license, users will need to obtain permission from the license holder to reproduce the material. To view a copy of this license, visit http://creativecommons.org/licenses/by/4.0/

C) The Author(s) 2017 